\title{
The Impact of Technology Trends on Healthcare Systems: A Study on Opportunities and Threats
}

\author{
Erdal Erdal \\ Assistant Professor, Department of Computer Engineering, \\ Kırıkkale University, Kırıkkale, Turkey
}

\begin{abstract}
The importance of health in human life is enormous. The technologies developed and the studies in this field have caused differences in the field of health as in all areas. The aim of this study is to examine the needs in the field of health and to set the criteria for determining the most appropriate technology. In the study, three new technology trends in literature are examined. The concepts of edge computing, fog computing and cloud computing were evaluated within the scope of the study. The literature of these three concepts has been examined and the areas where they can be preferred in the field of health services are presented. In addition, at the end of the study, the necessity of Futurist Healthcare systems, which will cover all health services, is stated.
\end{abstract}

Keyword: Healthcare, Information and communication technologies, Infrastructure, Cloud computing, Fog computing, Edge computing

\section{INTRODUCTION}

The importance of health in people's lives is undisputed. With the development of technology, great changes and developments have started in the field of health. Together with these changes, technology has taken its place among the indispensable elements of the health field. Applications and systems which are revolutionary in health have been developed. In this way, changes that have touched the lives of people have occurred. However, it is inevitable for the health sector to adapt to these developments with the advancing technology. In the technological field, classical and traditional data centers have been used decades ago. This phenomenon is still working and serving the technology infrastructure. However, with the deficiencies and technological developments, the traditional data centers have been replaced by different technological infrastructures. Traditional data centers nowadays appear to have been replaced by cloud computing, fog computing and edge computing. The main reasons underlying this need for change are listed below.

$>$ Increased amount of data to be stored

$>$ The need to share and transmit stored data

$>$ The requirement to analyze stored data

The aim of this study is to examine the technologies that can be used in health systems and health infrastructure. As a result of the examination, suggesting the most suitable infrastructure for the target will provide great improvements in health services.

However, prior to the determination of the infrastructure that is most suitable for the needs, it is necessary to identify the substructures that come with technological developments and to identify their advantages and disadvantages.

\section{LITERATURE REVIEW}

Before the detailed study of the studies to be carried out, it should be mentioned in the studies conducted in the past period in the literature.

In order to reveal the effects of technological developments on the health field clearly, the studies have been examined by giving a date range to technological concepts. At this stage, the surveys on the adaptation of Information and Communication Technologies (ICT) to the field of health services were evaluated in order to list more systematic and clear studies. 
It will not be wrong to start with cloud computing, which is the most commonly heard technology concept in this field. As seen in the literature studies, cloud computing is of great importance in the field of health services. In addition, cloud computing is still the preferred and used infrastructure [1-5].

Another popular technology developed in parallel with technological developments is the Internet of Things (IoT)[6].On the basis of IoT there is the creation of a global network structure with data from physical devices. In this way, it is aimed to connect the physical devices to each other via this network. Although it was initially restricted by radio frequency recognition (RFID) technology, the currently spoken IoT has reached different stages. Nowadays, when the concept of IoT is talked about, not only RFID technology, but also global positioning devices (GPS), mobile devices and all devices connected to the network actually come to mind[6].As mentioned before, no progress can be left unresponsive to technological developments, and the healthcare field has not been indifferent to this development. Studies in the field of healthcare services and studies on Iot are available in the literature [2, 7-11].

Another subject in the literature is big data. Today, the importance of large data has increased. One of the main reasons for this importance is the described IoT technology. Considering data from patients, doctors, diagnoses, treatments and hospitals in the field of health, the big data issue in this area is undisputed. Therefore, studies on the management of big data in the field of health have been conducted in the literature [2, 12-15].

In addition, there are a lot of concepts used in the field of healthcare with technological developments. Wireless body area networks(WBAN)[16], Wireless Sensor Network (WSN)[17], Machine-to-Machine communication (M2M)[8], Network technologies[2], 3D printing[18], robotics[19], social networks[20] and artificial intelligence[21] issues are out of scope.

In addition to cloud computing, there are solutions which are an alternative to cloud computing and the deficiencies in cloud computing are eliminated. The concepts of edge computing and fog computing will be studied in this study. There are also investigations and investigations on these issues in the health care literature [2, 22-24].
The concepts of cloud computing, which has become a phenomenon in this field with the fog computing and edge computing in the field of health services, have been determined as the scope of this study.

\section{TECHNOLOGY TRENDS}

Cloud computing, fog computing and edge computing topics will be examined in this heading. The architectures, advantages and disadvantages of these three concepts will be described. In order to select the most appropriate service type in the field of health, these concepts need to be detailed.

\section{A. Cloud Computing}

Cloud computing is the general name of Internetbased IT services for computers and other devices, providing computer resources that can be used at any time and shared between users[25].In the literature, there are studies related to cloud computing-based applications and services in the field of health services[3, 4, 25-27].

The studies in the literature have been examined and the highlights are listed are below [3, 4, 25-27].

$>$ Using cloud computing can develop and improve healthcare $\mathrm{C}$ sector, also bring important opportunities.

The transmission of all data over the internet causes concerns about the safety and confidentiality of the patient data and data management. Studies are still continuing in this area.

Cloud computing power can be used in decision support mechanisms in healthcare.

Cloud computing makes it easier to process big data analyzes in the health field.

High bandwidth requirements due to data transfers required.

Another hot topic in the literature is IoT and cloud computing is insufficient on this technology which is a big problem [28].

$>$ Cloud computing is a disadvantageous in terms of integrating with the types of services it provides such as Software as a Service (SaaS), Platform as a Service $(\mathrm{PaaS})$ and Infrastructure as a Service (IaaS)[29].

$>$ Storing files in the cloud in a distributed storage systems[30].

$>$ Difficulties in analyzing large data due to distributed file or data structure[31]. 


\section{B. Fog Computing}

Fog computing is the architecture that suggests that smart devices should be analyzed first at a local point and sent to central servers, as opposed to the architecture that allows the data to be sent and processed to a central server[32, 33].The approach based on fog computing is filtering, processing and storage operations by establishing an intermediate layer just before all data is kept on the cloud.

As of 2020, the number of IoT devices in the world is estimated to be 5.63K[34]. As can be seen in the estimates, it is certain that IoT will remain a hot topic for a long time. Developments in IoT technology will make this technology preferred in health sector as well. Therefore, it is imperative that the technological trend, which will be the subject of choice in the field of health, be in line with the IoT technology. In response to IoT problems in cloud computing, the proposed system provides solutions to IT problems.

For a clearer understanding of the concept of fog computing, an exemplary architecture from a study is shown in Figure 1[35].

Figure 1: Sample fog computing architecture[35]

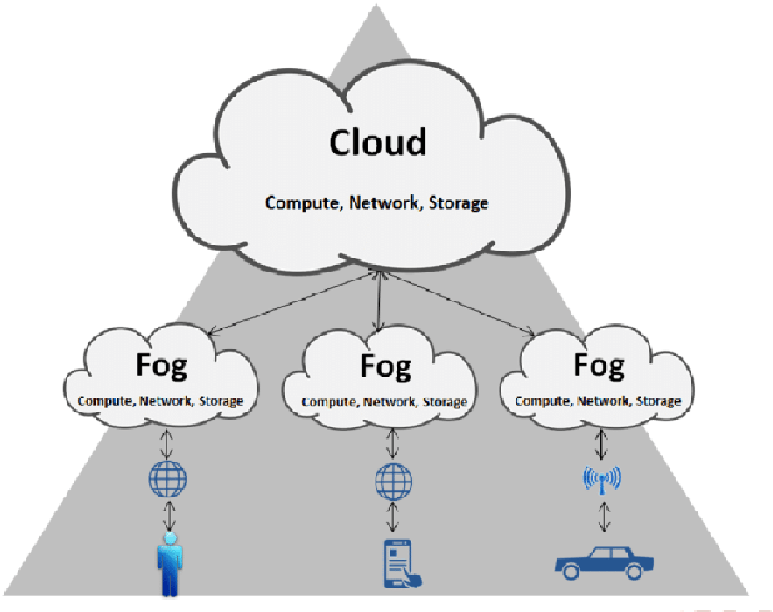

The studies about fog computing in the literature have been examined and the highlights are listed are below $[23,36,37]$.

$>$ Fog computing is suitable for real-time applications in healthcare services with low latency and high response time.

$>$ It is suitable for use with IoT technology used in healthcare services and applications.

$>$ It provides big data analysis with its processing power and storage space with its local architecture.

$>$ It offers better scalable architecture than cloud computing.
More powerful distributed processing thanks to local processing power.

> Safer and fault tolerant architecture thanks to local operations.

\section{Edge Computing}

Edge computing and fog computing have similarities because they provide closer data processing and data collection. However, although there are similarities between these two concepts, they have very important differences. The main difference between these two new technology trends is where the calculation and processing power are located. Sis computing includes processing on the local area network (LAN).In contrast; edge computing is based on the placement of computing and processing power into devices. In fact, fog computing uses edge devices over LAN.

The need for the development of edge computing is IoT technology, just as in the case of fog computing. In the literature, the studies related to edge calculation are examined and the important points are listed below $[22,38,39]$.

$>$ In e-healthcare or telemedicine applications and services, intensive patient data is received through wireless sensors. In such cases, edge computing can respond to this need.

It is fully compatible and suitable for IoT technology.

Cost is higher than cloud computing.

$>$ It is suitable to be preferred in applications with low latency period.

$>$ It is required to have the storage capacity and processing capacity of the devices it contains.

\section{SUGGESTIONS}

The impact of technology trends on healthcare systems has been examined. Cloud computing, fog computing and edge computing fields, which are one of the newest trends in technology, have been included in the study. Apparently it is not possible to talk about a single technology trend for every health system. Therefore, health areas are categorized according to their needs.

As a result of this study, the following suggestions have been presented.

\section{A. Traditional Health Systems}

Cloud computing systems meet the needs of today's traditional health systems. Cloud computing systems are inherently more cost effective than traditional data centers. Intensive data flow is available in the systems 
used in health services. Therefore, the bandwidth requirement of this system is enormous. In the health care system, patient records, that is, confidential information about the patients are available. Storing these data in the cloud brings to mind the safety issue. It is stated that there are studies in the literature on this subject.

\section{B. IoT Based Health Systems}

IoT is one of the new trends of the technology. IoT studies are also carried out in the field of health services. The IoT technology is inherent in its logic and it receives data from all edge devices. Given the data flowing from these devices, the amount of data reaches enormous dimensions. The use of edge computing in such health applications is considered appropriate. However, at all edge points, data processing and storage needs cost.

\section{Real-Time Application Based Health Systems}

Using cloud computing in health systems where realtime services are available may not be considered very appropriate as it may cause loss of time and bandwidth compression. Real-time applications are critical systems that are inherently in need of immediate response. In such systems, fog information or edge computing can be preferred according to more detailed needs. In this way, it is possible to transfer data independently from internet and bandwidth.

\section{Futurist Healthcare Systems}

The issue mentioned in this category is to provide the most optimal solution covering all health systems. The targeted solution should include both fog computing, edge computing and cloud computing based on the infrastructure needed. Real-time applications can communicate via cloud computing, traditional health applications through fog and edge computing. However, there is a need for an entire architecture and framework that can meet this need.

\section{CONCLUSION}

In this study, new technology trends in health services systems and applications are examined and recommendations are presented. Developing technology affects all areas of our lives. However, every technological trend and concept may not be good or appropriate for every application. Therefore, fog, edge and cloud computing technologies which can be used in health system are examined and suggestions are presented as a result of examinations. A useful research has been introduced for new systems to be developed and new applications to be developed in the field of health. It was also determined that there was a need for a framework where all these technologies were brought together.

\section{REFERENCES}

1. Abbas, A. and S.U. Khan, A review on the stateof-the-art privacy-preserving approaches in the ehealth clouds. IEEE J Biomed Health Inform, 2014. 18(4): p. 1431-41.

2. Aceto, G., V. Persico, and A. Pescapé, The role of Information and Communication Technologies in healthcare: taxonomies, perspectives, and challenges. Journal of Network and Computer Applications, 2018. 107: p. 125-154.

3. Calabrese, B. and M. Cannataro, Cloud Computing in Healthcare and Biomedicine. Scalable Computing: Practice and Experience, 2015. 16(1).

4. Ermakova, T., et al. Cloud Computing in Healthcare - a Literature Review on Current State of Research. in Proceedings of the Nineteenth Americas Conference on Information Systems. 2013. Chicago, Illinois.

5. Pino, C. and R.D. Salvo. A Survey of Cloud Computing Architecture and Applications in Health in Proceedings of the 2nd International Conference on Computer Science and Electronics Engineering. 2013. Paris, France: Atlantis Press.

6. Qi, J., et al., Advanced internet of things for personalised healthcare systems: A survey. Pervasive and Mobile Computing, 2017. 41: p. 132-149.

7. Darshan, K.R. and K.R. Anandakumar, A comprehensive review on usage of Internet of Things (IoT) in healthcare system. 2015: p. 132136.

8. Geng, W., et al., M2M: From mobile to embedded internet. IEEE Communications Magazine, 2011. 49(4): p. 36-43.

9. Laplante, P.A. and N.L. Laplante, A Structured approach for describing healthcare applications for the Internet of Things. 2015: p. 621-625.

10. Riazul Islam, S.M., et al., The Internet of Things for Health Care: A Comprehensive Survey. IEEE Access, 2015. 3: p. 678-708.

11. Yeole, A.S. and D.R. Kalbande, Use of Internet of Things (IoT) in Healthcare. 2016: p. 71-76.

12. Archenaa, J. and E.A.M. Anita, A Survey of Big Data Analytics in Healthcare and Government. Procedia Computer Science, 2015. 50: p. 408-413.

13. Ramesh, D., P. Suraj, and L. Saini, Big data analytics in healthcare: A survey approach. 2016: p. 1-6. 
14. Sun, J. and C.K. Reddy, Big data analytics for healthcare. 2013: p. 1525.

15. Zou, Q., et al., Survey of MapReduce frame operation in bioinformatics. Brief Bioinform, 2014. 15(4): p. 637-47.

16. Filipe, L., et al., Wireless Body Area Networks for Healthcare Applications: Protocol Stack Review. International Journal of Distributed Sensor Networks, 2015. 2015: p. 1-23.

17. Ko, J., et al., Wireless Sensor Networks for Healthcare. Proceedings of the IEEE, 2010. 98(11): p. 1947-1960.

18. Malik, H.H., et al., Three-dimensional printing in surgery: a review of current surgical applications. J Surg Res, 2015. 199(2): p. 512-22.

19. Ciuti, G., et al., Frontiers of robotic endoscopic capsules: a review. J Microbio Robot, 2016. 11(1): p. 1-18.

20. Griffiths, F., et al., Social networks--the future for health care delivery. Soc Sci Med, 2012. 75(12): p. 2233-41.

21. Kassahun, Y., et al., Erratum to: Surgical robotics beyond enhanced dexterity instrumentation: a survey of machine learning techniques and their role in intelligent and autonomous surgical actions. Int J Comput Assist Radiol Surg, 2016. 11(5): p. 847.

22. Chen, M., et al., Edge cognitive computing based smart healthcare system. Future Generation Computer Systems, 2018. 86: p. 403-411.

23. Kumari, A., et al., Fog computing for Healthcare 4.0 environment: Opportunities and challenges. Computers \& Electrical Engineering, 2018. 72: p. 1-13.

24. Rahmani, A.M., et al., Exploiting smart e-Health gateways at the edge of healthcare Internet-ofThings: A fog computing approach. Future Generation Computer Systems, 2018. 78: p. 641658.

25. Ali, O., et al., Cloud computing-enabled healthcare opportunities, issues, and applications: A systematic review. International Journal of Information Management, 2018. 43: p. 146-158.

26. Al-Ruithe, M., E. Benkhelifa, and K. Hameed, Current State of Cloud Computing Adoption - An Empirical Study in Major Public Sector Organizations of Saudi Arabia (KSA). Procedia Computer Science, 2017. 110: p. 378-385.

27. Bayramusta, M. and V.A. Nasir, A fad or future of IT?: A comprehensive literature review on the cloud computing research.International Journal of
Information Management, 2016. 36(4): p. 635644.

28. Díaz, M., C. Martín, and B. Rubio, State-of-theart, challenges, and open issues in the integration of Internet of things and cloud computing. Journal of Network and Computer Applications, 2016. 67: p. 99-117.

29. Souri, A., N.J. Navimipour, and A.M. Rahmani, Formal verification approaches and standards in the cloud computing: A comprehensive and systematic review. Computer Standards \& Interfaces, 2018. 58: p. 1-22.

30. Leavitt, N., Storage Challenge: Where Will All That Big Data Go? Computer, 2013. 46(9): p. 2225.

31. Talia, D., Clouds for Scalable Big Data Analytics. Computer, 2013. 46(5): p. 98-101.

32. Aazam, M., S. Zeadally, and K.A. Harras, Offloading in fog computing for IoT: Review, enabling technologies, and research opportunities. Future Generation Computer Systems, 2018. 87: p. 278-289.

33. Zhang, P., M. Zhou, and G. Fortino, Security and trust issues in Fog computing: A survey. Future Generation Computer Systems, 2018. 88: p. 1627.

34. Research, G.V., Fog Computing Market Analysis By Solution, By Hardware, By Application, By Region, \& Segment Forecasts, 2018 - 2025. 2017. p. 100.

35. Al Hamid, H.A., et al,, A Security Model for Preserving the Privacy of Medical Big Data in a Healthcare Cloud Using a Fog Computing Facility With Pairing-Based Cryptography. IEEE Access, 2017. 5: p. 22313-22328.

36. Mutlag, A.A., et al., Enabling technologies for fog computing in healthcare IoT systems. Future Generation Computer Systems, 2019. 90: p. 6278.

37. Yassine, A., et al., IoT big data analytics for smart homes with fog and cloud computing. Future Generation Computer Systems, 2019. 91: p. 563573.

38. Alam, M.G.R., et al., Edge-of-things computing framework for cost-effective provisioning of healthcare data. Journal of Parallel and Distributed Computing, 2019. 123: p. 54-60.

39. Olaniyan, R., et al., Opportunistic Edge Computing: Concepts, opportunities and research challenges. Future Generation Computer Systems, 2018. 89: p. 633-645. 